\title{
Narrative review of immunotherapy in thymic malignancies
}

\author{
Jose Carlos Benitez ${ }^{1}$, Benjamin Besse ${ }^{1,2}$ \\ ${ }^{1}$ Gustave Roussy, Department of Cancer Medicine, Villejuif, France; ${ }^{2}$ Université Paris-Saclay, Orsay, France \\ Contributions: (I) Conception and design: JC Benitez; (II) Administrative support: B Besse; (III) Provision of study materials or patients: JC Benitez; \\ (IV) Collection and assembly of data: JC Benitez; (V) Data analysis and interpretation: All authors; (VI) Manuscript writing: All authors; (VII) Final \\ approval of manuscript: All authors. \\ Correspondence to: Benjamin Besse, MD, PhD. University Paris-Saclay and Gustave Roussy Cancer Campus, 114 Rue Edouard Vaillant, 94805 \\ Villejuif, France. Email: Benjamin.BESSE@gustaveroussy.fr.
}

\begin{abstract}
Thymomas and thymic carcinomas (TCs) (also known as Thymic Epithelial Tumors or TETs) are rare cancers and the most frequent masses of the anterior mediastinum. These tumors appear in the epithelial component of the thymus, a primary lymphoid organ, and they have reported a high risk of autoimmunity due to a unique biology. Indeed, up to $30 \%$ of patients with TETs could present an autoimmune disorder (AID), the most frequent being Myasthenia Gravis (MG). Moreover, AIDs have been reported not only at tumor diagnosis but before and during the follow-up. These tumors have a lack of specific therapeutic targets for metastatic setting. Immune checkpoint inhibitors (ICI) may defeat cancer cells' capacity to evade the immune system and proliferate. The long-term benefit of ICIs in the metastatic setting in several tumors, such as melanoma or non-small cell lung cancer (NSCLC), let to evaluate ICI approaches in TETs. The high rate of AIDs and distribution of autoimmune events among TET's histological subtypes may have an influence on the decision regarding a treatment based on ICI due to the increased risk of toxicity. We summarize the current evidence for the efficacy of ICI in thymoma and TC and discuss several unresolved challenges and concerns for the use of this agents in TETs.
\end{abstract}

Keywords: Thymic epithelial tumors (TETs); immune checkpoint inhibitors (ICI); autoimmune disorders (AIDs); treatment related toxicity

Submitted Dec 01, 2020. Accepted for publication Jun 07, 2021.

doi: $10.21037 /$ tlcr-20-1222

View this article at: https://dx.doi.org/10.21037/tlcr-20-1222

\section{Introduction}

Thymic epithelial tumors (TETs) are rare and potentially aggressive malignant cancers of the anterior mediastinum (1). A low Incidence (1.3 and 1.7/million inhabitants per year) has been reported with around 1,500 cases in Europe $(2,3)$ and a mean age at diagnosis of 40 to 50 years (2). The World Health Organization (WHO) differentiates two entities, thymoma and thymic carcinoma (TC) (4) based on the rate and morphology of the two components of the thymic gland, lymphocytes and epithelial cells. The epithelial component is the only one that could develop cancer. At the International Thymic Malignancy Interest Group (ITMIG) consensus meeting in 2011 (5), the WHO classification was validated as the standard for clinical practice (6) defining six main histological subtypes according to proportion of non-malignant appearing thymic epithelial cells and the rate of lymphocytes (7) (thymomas A, AB, B1, $\mathrm{B} 2$ and $\mathrm{B} 3$ ). Less common than thymoma, TC comprises approximately $10-15 \%$ of TETs and are highly associated with a poor prognostic and the development of distance metastases (1), the most common histology assessed in this subgroup of TETs is squamous cell carcinoma. TC harbors phenotypes expression of CD5 and CD117/KIT by immunohistochemistry (IHC) and reports specific molecular features that differs from thymomas and from squamous cell lung cancer (8).

Regarding the clinical setting, TETs could appear as encapsulated tumors or stage I based on surgical classification by Masaoka and Koga (MK), 65\% of cases, 


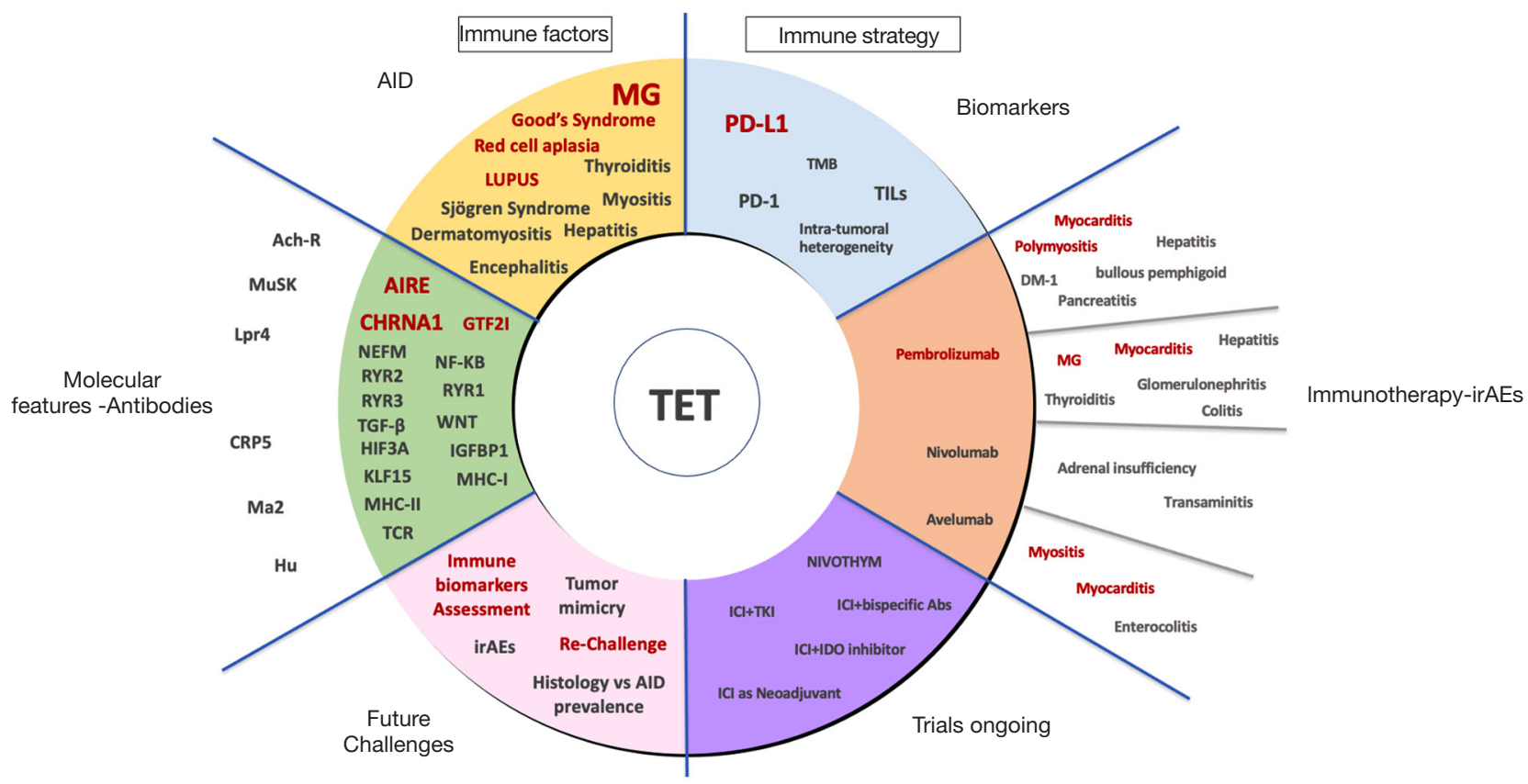

Figure 1 Graphic representation of TETs' immune factors and strategies. The use of immune checkpoint inhibitors (ICIs) for patients with TETs remains a challenge. The high rate of AIDs and the lack of proved biomarkers to select the patients make difficult the inclusion of patients within clinical trials. Several studies of TETs and AID landscape have been performed but no solid results were reported. Aggressive histological subgroups (thymoma B3 and TC) have been treated with ICI, with noted toxicity; several clinical trials are currently ongoing.

or invasive tumors (stages II-IV in the MK classification, $35 \%$ of cases) (9-11). Surgery represents the keystone step for the curative-intent treatment in MK I/II and some stage III tumors, as complete resection represents the most important prognostic factor in terms of survival $(12,13)$, in addition to MK stage (10) and histological features (14), for which a correlation has been found (15). However, between $8 \%$ and one third of patients reporting a thymoma or $25 \%$ to $59 \%$ of TCs will present a recurrence depending on MK surgical classification (14,16-18). Platinum-based combination chemotherapy regimens are the standard treatment for metastatic, unresectable or refractory disease although options are limited in this setting (16) with response rates ranging from $69 \%$ in thymoma to $42 \%$ in TC (19). Furthermore, the efficacy of other targeted therapies such as sunitinib (20) and everolimus (21) has been demonstrated. Exceptionally, others targeted pathways such as $c-K I T$ and PI3KCA genes as well as epigenetic pathways have been explored (22-24). The estimated five years OS is $80 \%$, and $40 \%$ for thymoma and TC respectively (25).

ICIs have changed the paradigm of cancer care becoming the standard treatment for several tumor types such as melanoma (26), lung cancer (27) or, bladder cancer (28). Their role is not clear for TETs due to the high frequency of autoimmunity leading a high risk of toxicity. This review aims to show the available evidence in this setting and the potential challenges with related autoimmune disorders (AIDs) and possible predictive biomarkers (Figure 1). We present the following article in accordance with the narrative review reporting checklist (available at https:// dx.doi.org/10.21037/tlcr-20-1222).

\section{Autoimmunity and tumors of the thymus}

\section{Thymic physiology and pathogenesis in TETs}

The thymic gland is a keystone for the development of immune tolerance. After having been generated in the bone marrow, immature thymocytes ( $\mathrm{T}$ cell progenitors) undergo maturation in the thymus through interactions with cortical and medullary thymic epithelial cells (mTECs). The presentation of tissue-specific self-antigens (TSAs) through major histocompatibility complex class II (MHC- 


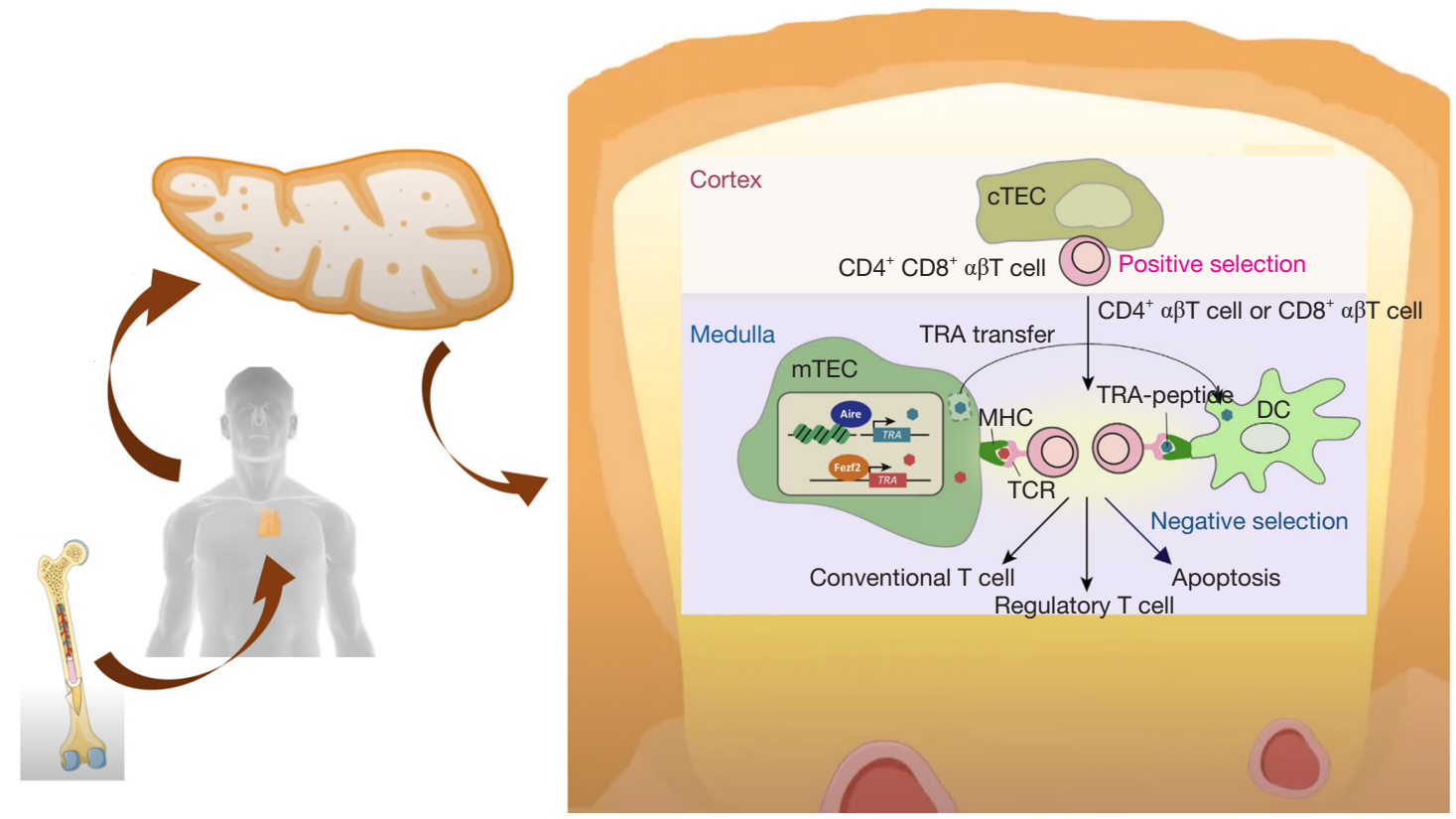

Figure 2 Schematic diagram of positive and negative $T$ Cell selection in the thymus. Immature thymocytes leave the bone marrow and undergo maturation in the thymus through interactions with cortical and medullary thymic epithelial cells. Autoreactive $\mathrm{T}$ cells are negatively selected in the medulla through mTEC expression of self-antigens such as tissue-restricted antigens (TRAs). TRAs are promiscuously expressed under the control of the autoimmune regulator Aire, but also regulated by the transcription factor Fezf2 in mTECs. Adapted from: Takaba, H., \& Takayanagi, H. Trends in Immunology, 2017 (31).

II) is regulated by two transcription factors, autoimmune regulator (AIRE) and Fez family zinc finger 2 (Fezf2) genes $(29,30)$. The passage through the thymic cortex and corticomedullary junction of $\mathrm{T}$ thymocytes implies phenotypic modifications resulting in a functioning $\mathrm{T}$ cell. Immature $\mathrm{T}$ cells reacting with MHC-II are able to enter the thymic medulla whereas those with no interaction are eliminated. In the medulla of the thymus, we can find both dendritic cells and mTECs, those mTECs expressing AIRE suffer different changes and undergo apoptosis, setting free TSAs to dendritic cells of the thymus. T cells reacting against TSAs also undergo apoptosis carrying out the immune tolerance (29) (Figure 2). This phenomenon occurs mainly during childhood but it is still present in adults (32) and could be deregulated along with thymic carcinogenesis.

In TETs, the normal thymic structure is altered with abnormal thymic epithelial cells, downregulation of MHC class II and, of AIRE expression. AIRE has a unique capability to express all TSAs at mTECs cell surface. The defusing of the gene AIRE leads to the absence of expression for some TSAs and the release of self-reactive lymphocytes out of the thymus resulting in an increased predisposition towards development of AIDs $(33,34)$. Moreover, autoreactive $\mathrm{T}$ cells modify self-antigens expression on TETs' cells liberating interferon-gamma $(I F N-\gamma)$ so, upregulating PD-L1 expression in tumor cells (35). These findings explain the high tumor cell PD-L1 expression in thymic tumors, up to $92 \%$ and $100 \%$ in thymoma and TC respectively (36). This rationale supports the use of Immune Checkpoints Inhibitors (ICIs) in TETs although it must be balanced with the increased risk of treatment-related adverse events (TRAEs).

\section{AIDs in TETs}

Autoimmune events must be differentiated from paraneoplastic syndromes. Their pathophysiology, clinical management and survival outcomes are different. Paraneoplastic disorders generally result from production of hormones, cytokines or peptides by tumor cells leading to metabolic derangements and peripheral induction of autoantibodies produced by tumor cells (37). Thus, successful treatment of the tumor should improve paraneoplastic symptoms whether AIDs may rest beside a 
radical treatment due to a deregulation in the physiological role of the thymus and faulty selection of immature $\mathrm{T}$ cells, as meant above.

AIDs are present in more than $30 \%$ of TETs $(38,39)$. The most common syndrome is myasthenia gravis (MG) (38), which can vary from $17 \%$ in thymoma $\mathrm{A}$ to $71 \%$ in thymoma B2 (13). In addition, endocrine, rheumatologic, gastrointestinal, renal, cutaneous affectations may be associated (40-42). Data from RYTHMIC (Réseau tumeurs THYMiques et Cancer), one of the biggest registries of TETs in Europe, showed a 20.2\% (590/2,909) of AID prevalence with $3.8 \%(n=21)$ of the patients presenting more than one event. Most of patients had MG (69.6\%), followed by Good's syndrome (5.6\%), pure red cell aplasia $(2.8 \%)$, thyroiditis $(3.4 \%)$ and systemic erythematous lupus (4.4\%). The AIDs were presented before $(12.6 \%)$ and after $(6.4 \%)$ besides the diagnosis (81\%). Concerning histologic subtypes, more than $40 \%$ of prevalence were assessed in B2 and B3 subtypes ( $45 \%$ and $41 \%$ respectively) $(43,44)$. Thymomas $\mathrm{AB}, \mathrm{B} 1$ and $\mathrm{B} 2$ are rich with lymphocyte agglomerates, suggesting that those tumors are most frequently associated with AIDs than TC and B3 thymomas (45). However, several series have found a high prevalence of autoimmune events in thymoma B3 $(13,14,43)$. Importantly, TC are rarely associated with AIDs $(41,46)$. Several series have reported no MG in TC $(14,47)$ with a small representation of AIDs overall $(44)$ maybe due to the presence of non-immature T-cells. The high incidence of AIDs in TETs in addition of the presentation of autoimmune symptoms afterward diagnosis of cancer $(43,44)$ requires an extremely close monitoring during treatment with ICI.

\section{Molecular features of AIDs and TETs}

The unique biology of TETs confers them a special interest for the study of autoimmunity due to the strong association with AIDs, especially with MG. Indeed, the knowledge about the molecular characteristics of thymoma-related MG (TRMG) is limited. It is well understood that antibodies against acetyl-choline receptor (AchR) are essential for the development of MG, antiAchR can block the post-synaptic membrane as well as reduce the quantity of AchR at the neuromuscular junction leading to a decrease response to Ach, thus a fluctuating skeletal muscle weakness and fatigue (48). Immunoregulation deficiency and central tolerance induced by tumor microenvironment may be a cause for the pathogenesis of tumor-associated AIDs, such as MG.

Several studies have investigated whether different subunits of Ach are expressed in the thymus and if some are associated with the development of TRMG (49). Low levels of AIRE and AchR expression by tumor cells have been associated with higher risk of developing MG (50). Moreover, relative RNA expression levels of Foxp3 were significantly higher in tissue samples from patients without AIDs compared to those suffering MG and/or other AIDs (50). Of note, AIRE and Foxp3 are transcription factors with an important role in T-reg lymphocytes differentiation, which have an important role to downregulate autoimmunity, but also can promote tumor growth $(50,51)$. Interestingly, AIDs can be associated with specific genomic alterations such as NF-KappaB/AIRE pathway deregulation, related with TRMG (52). One of the largest molecular studies concerning TETs have been made by the TCGA, Radovich and colleagues analyzed 117 TETs reporting a higher rate of aneuploidy in thymomas from patients reporting MG (22). Furthermore, some genes have been correlated to MG; expression levels of the Ach-R $\alpha$-subunit gene (CHRNA1) was higher in samples from patients with TRMG. In addition, the mediumsized neurofilament (NEFM), with similar immunogenic properties of its protein with the AChR $\alpha$-subunit (53) and titin (54), was mainly overexpressed in thymomas A and $\mathrm{AB}$ subgroups showing TRMG, while types $\mathrm{B} 1 / \mathrm{B} 2$ and $\mathrm{B} 3$ thymomas overexpressed the neuronal RYR3, with likeliness to muscular RYR1 and cardiac RYR2 (22). Regarding TC, with more aggressive behavior, several tumor suppressors including CYLD, CBFB, CDH1, CDH11, CTCF, and ZFHX3 was found, as well as a higher Tumor Mutational Burden (TMB) compared to thymomas (22). Those findings support the hypothesis that TC and thymoma are distinguished by their genetic and epigenetic profiles. Indeed, recent results of a French study after a huge transcriptomic analyses of 2,560 genes in 194 TETs samples are in this line. The authors found two different clusters of genes differentiating TC from thymoma (55).

A Chinese study with a cohort of 105 patients reporting and not MG found that patients suffering MG showed elevated inflammatory responses and metabolic related pathways, whereas those patients with no autoimmune event were presented with mesenchymal characteristics (56). Of note, GTF2I mutations were assessed at significantly higher frequency in patients with no autoimmunity (56). Indeed, GTF2I mutation have been correlated with better survival outcomes (57); thus, we could hypothesize that 
patients with GTF2I alterations would be good candidates for immunotherapy; however, GTF2I mutations have been detected in type $\mathrm{A}(82 \%)$ and type $\mathrm{AB}(74 \%)$ and rarely in the aggressive subtypes $(22,57)$, such as $\mathrm{TC}$, where recurrent mutations of known cancer genes have been identified including TP53, CYLD, CDKN2A, BAP1 and PBRM1 (22,57). Highlighting, GTF2I have been associated to severe toxicity to immunotherapy (58). Therefore, further analysis regarding GTF2I as a biomarker are warrant.

Some other molecular features have been further described for patients not reporting TRMG, such as upregulated TGF- $\beta$ and WNT pathways (56), nonetheless those routes hardly respond to immunotherapy. Finally, several other biomarkers have been evaluated to differentiate thymomas from TCs $(55,59)$ and to understand the pathogenesis of MG such as IGFBP1, KLF15, PDK4 and, HIF3A (60). Indeed, other AIDs such as encephalitis or polymyositis has been correlated with the enhance of anti$\mathrm{Hu}$ antibodies, Ma2 antibodies and CRP5 antibodies (61) or deregulation of T-cell-receptor (TCR) and upregulated MHC-I expression on muscle fibers (62); but MG and AID landscape is still unknown.

\section{Immune strategy in TETs}

In recent years, ICIs have revolutionized treatment strategies and prognosis of several solid tumors. In pre-treated patients, ICI yielded 5 -year OS of $34 \%$ in melanoma, $28 \%$ in renal cell carcinoma, and $16 \%$ in nonsmall cell lung cancer (NSCLC) (26), leading to approval of anti-Programmed cell Death protein 1 inhibitor (anti-PD1), anti-Programmed Death Ligand 1 inhibitor (anti-PDL1), and anti-Cytotoxic T Lymphocyte Antigen 4 inhibitor (anti-CTLA4) for metastatic disease in several tumors. Considering the long-term benefit of ICI in many solid tumors, these and other immune related strategies have been proposed with hope for patients with TETs to derive similar meaningful benefit.

\section{Immune-related and predictive biomarkers}

Several biomarkers have been analyzed as predictors of ICI efficacy, nonetheless only two have been stablished as biomarkers of response for ICI: (I) PD-L1 expression in tumor cells and, (II) Tumor Mutational Burden (TMB) that shows the number of non-synonymous single nucleotide variants in a tumor genome coding area. Tumors with high TMB have more neoantigens enhancing immune response thus leading to better efficacy of ICI as it was shown in previous studies $(63,64)$. Recently, intra-tumoral heterogeneity has been described as a good predictive factor for immunotherapy outcomes in melanoma, in fact high intra-tumoral heterogeneity yields better responses to ICIs compared to those developed from a single clone (65). Further, for adequate activation of immune system a high presence of tumor-infiltrating lymphocytes (TILs) in tumor tissue is required to reach better treatment response (66).

Expression of PD-L1 have been observed in more than $90 \%$ of epithelial cells of the normal thymus (67) and have been widely evaluated in TETs being assessed in thymoma and TC with direct relation with histology aggressiveness. High expression of PD-L1, IDO and FOXP3+ Tregs have been associated with a higher grade of tumor histology in patients with TC in a large series of 100 thymomas and 69 TC (68). In other studies PDL1 expression ranged, in thymoma, from $23 \%$ to $92 \%$ of tumor cells, and in TC, from $36 \%$ to $100 \%$ of tumor cells (36,67,69-73). This upregulated expression of PD-L1 in TETs may be led by IFN- $\gamma$ presence in TETs' cells (35). Indeed, clinical and pathological elements have been correlated with high PD-L1 expression, such as young age, advanced MK stage, no complete resection and history of neoadjuvant therapy for thymoma (74). By contrast, correlation with histology subgroups remains unclear $(70,71,75)$. Actually, there are no solid results regarding survival outcomes considering that some of the studies have correlated a high PD-L1 expression with better survival $(71,74)$ but others with poor outcomes (70). Furthermore, TILs have been analyzed besides PD-L1 although poor evidence is available. Higuchi and colleagues reached PD-L1 and TILs in surgical samples from 39 patients with thymomas and TC. PD-L1 higher than $1 \%$ was reported in $54 \%$ of the specimens with no same distribution among TETs' subgroups $(\mathrm{B} 2>\mathrm{B} 3>\mathrm{TC}>\mathrm{B} 1>\mathrm{AB}>\mathrm{A})$. High $\mathrm{CD} 8+(84 \%)$ among CD3+ TILs infiltration was assessed and diffusely distributed in all cases (73). High PD-1 expression in TILs have been found in $23 \%$ to $62 \%$ of TCs as well, with no prognostic nor predictive correlation $(36,75)$. Interestingly, TETs TMB has been demonstrated to be one of the lowest among tumors (22). Whether PD-L1 is the best predictive biomarker remains controversial due to the impairment of many patients notwithstanding the use of ICI. More beneficial treatment effects seem better for aggressive thymomas such as type B2 or B3 although 
Table 1 Clinical trials with immune checkpoint inhibitors in thymic epithelial tumors

\begin{tabular}{|c|c|c|c|c|c|c|c|}
\hline Author & Phase & Treatment & $\mathrm{N}$ & RR/DCR (\%) & PFS (mo) & OS (mo) & irAEs $G \geq 3(\%)$ \\
\hline \multirow[t]{2}{*}{ Cho et al. (77) } & II & Pembrolizumab & $26 \mathrm{TC}$ & $19 / 73 \%$ & $6.1 \mathrm{mo}$ & $14.5 \mathrm{mo}$ & $15.4 \%$ \\
\hline & & & $7 \mathrm{~T}$ & $29 / 100 \%$ & & NR & $71.4 \%$ \\
\hline Katsuya et al. (78) & II & Nivolumab* & $13 \mathrm{TC}$ & $0 / 38 \%$ & $3.8 \mathrm{mo}(1.9-5.6)$ & Nt R (11.3-NA) & $13 \%$ \\
\hline Heery et al. (79) & & & $1 \mathrm{TC}$ & & & & \\
\hline
\end{tabular}

T, thymoma; TC, thymic carcinoma; TRAE, treatment-related adverse events. $\mathrm{G} \geq 3$ : Grade $\geq 3$. Mo, months; NR, not reached; Nt R, not reported. *, study was closed prematurely and no responses were found. Note: In Giaccone et al. trial, CT-scans were performed every 6 weeks, whereas in Cho et al. trial every 9 weeks.

the high prevalence of AIDs aware the difficulty for the use of ICI.

\section{ICIs efficacy in clinical trials}

ICIs have been assessed in several clinical trials in TETs. Giaccone and colleges carried out a single-arm phase II study where patients with recurrent TC were treated with pembrolizumab (a fully humanized IgG4 antibody targeting PD-1 receptor). Patients with history of AIDs were excluded from this trial. Among 40 evaluable patients, an overall response rate (ORR) of $22.5 \%$ was observed. Disease control was achieved in 30 (75\%, 95\% CI, 59-87\%) patients with a median duration of response of 3 years. Median progression-free survival (mPFS) was 4.2 months (95\% CI, 2.9-10.3) and mOS was 24.9 months (95\% CI, 15.5-not reached). One-year PFS and OS were 29\% and $71 \%$, respectively and, five-year survival of $8 \%$. Ten (25\%) patients presented a high PD-L1 expression in tumor cells (at least 50\%), it was associated with longer survival (mPFS 24 vs. 2.9 months; mOS not reached vs. 15.5 months); only three of the 27 patients with PD-L1 expression by tumor cells less than $50 \%$ had a response. IFN- $\gamma$ signature assessed using the Nanostring assay correlated with response to pembrolizumab, as well as TP53 mutations, reached in the $36 \%$ of tumors, were associated to lower expression of PD-L1 and shorter OS. Interestingly, 4 patients were rechallenged with pembrolizumab after relapse with 2 responses to the treatment, one of them after 2 years completion therapy with pembrolizumab $(58,76)$ (Table 1$)$.

Cho et al. conducted a second clinical trial with similar design evaluating pembrolizumab in 26 patients with recurrent TC and in 7 patients with recurrent thymoma (four subtype B1, one subtype B2/B3 and one subtype B3). Three patients had a history of MG, but patients with active AID under systemic treatment or suffering severe AIDs were excluded. The ORR was $19.2 \%$ in patients with TC and $28.6 \%$ in patients with thymoma. Likewise, of 26 patients with TC, 5 (19\%) had a partial response and 14 (54\%) had stable disease. Tumors with high PD-L1 were more likely to respond to treatment. The median duration of response was not reached in patients with thymoma and was 9.7 months (95\% CI, 0.0-19.8) in patients with TC. mPFS was 6.1 months in both groups (95\% CI, 4.3-7.9 and 5.1-7.1 for thymoma and TC, respectively). Median OS was 14.5 months for TC and not reached in patients with thymoma (77) (Table 1).

Avelumab (a fully human IgG1 anti-PD-L1 antibody) was evaluated in 7 and 1 patients diagnosed of thymoma and TC with no history of autoimmune disease, respectively. Among the thymomas, 2 patients with subtype B3, 1 with subtype B2/B3, 2 with subtype B2 and 1 with subtype B1 were reported. Four of 7 patients (57\%) with thymoma had an objective response including a confirmed partial response in $2(29 \%)$ patients. Of note, significant tumor shrinkage was observed after one dose of avelumab in three patients $(79,80)$ (Table 1).

Finally, a phase II Japanese trial assessed the role of nivolumab (IgG4 antibody that targets the PD-1 receptor) in the treatment of patients with unresectable or recurrent TCs. Of the 15 patients included, 11 patients had stable disease, including five patients for 24 or more weeks. Median PFS and median OS was 3.8 months (95\% CI, 1.9-7.0) and 14.1 months (95\% CI, 11.1-not-reach), respectively. Patient accrual was terminated during the first 
stage due to none of the patients reached a response (78) (Table 1).

\section{Increase of Autoimmune toxicity in clinical trials}

Activation of the immunity increases the risk of developing immune-related adverse events (irAE). The incidence of TRAE in these trials is relatively high compared with the results of ICIs in other pathologies like melanoma, non-small-cell lung cancer, head and neck squamous cell carcinoma and urothelial carcinoma, where an incidence of grade $\geq 3$ irAE range between $3 \%$ and $9.7 \%$ have been assessed (81).

Among the 40 patients treated with pembrolizumab by Giaccone and colleges, 6 (15\%) developed serious AIDs reporting two cases of polymyositis and myocarditis; one case of pancreatitis, hepatitis and type 1 diabetes mellitus; one case of bullous pemphigoid; one case of polymyositis and hepatitis; and one case of transaminitis. Three patients had to discontinue treatment due to the toxicity. Patients affected by myocarditis and polymyositis and the one with bullous pemphigoid needed steroids to recover $(58,76)$. Of note, one of the patients who reported myositis, myocarditis and MG was under complete response for 40 months and $10 \%$ of patients shown more than one severe irAEs (76). In the Korean study, 5 (71\%) of seven patients with thymomas and four (15\%) of 26 patients with TC reported grade 3 or greater irAEs including hepatitis $(12.1 \%)$, myocarditis (9.1\%) and MG (6.1\%) (some the patients had previous history of MG); in addition, other patients presented thyroiditis, antineutrophil cytoplasmic antibody-associated rapidly progressive glomerulonephritis, colitis and subacute myoclonus. Eight patients $(24.2 \%)$ discontinued the treatment. Treatment of these toxicities was mainly based on steroids and immunoglobulins (77). Moreover, only two patients treated with nivolumab presented with severe AID (elevated transaminases and adrenal insufficiency (78). Patients under treatment with avelumab reported TRAEs in more than $15 \%$ of the cases (all grades) which were potential irAEs in $5(63 \%)$ patients. All responders reported irAEs, myositis in 3 patients (all after 1 dose of avelumab) and enteritis in 1 patient $(79,80)$ (Table 1).

The incidence of myocarditis in these trials is a concern, as myocarditis is associated with TETs in less than $1 \%(34)$. Myocarditis was observed in 5\% patients with TC and $43-57 \%$ patients with thymoma enrolled in clinical trials under treatment with ICIs $(58,77,79)$. This fact has also been assessed in several case reports of the use of ICIs in
TETs $(82,83)$. Myositis was observed in $8 \%$ of patients with TC treated with pembrolizumab and more than half of thymoma patients enrolled on a dose-escalation trial of avelumab $(58,80)$. Toxicity reported at the muscle could be explained due to the existence of increased TCR clones and upregulated MHC-I expression on muscle fibers with inflammatory infiltrates of macrophages and lymphocytes after treatment with ICI; of note, patients who developed myositis had no specific antibodies before and after ICI treatment (84). More frequent in TETs, up to $30 \%$ (38), MG have been reported as an irAE in $3-14 \%$ of TET patients treated with pembrolizumab $(58,77)$. Antibodies against the AchR are essential for the development of MG, as explained above, but also MuSK and Lrp4 antibodies (85). Interestingly, pure red cell aplasia has been described as the most common AID besides MG (43), however it has not been reported in these trials with ICI.

\section{Ongoing trials}

Immunotherapy is not evoked in type B1/B2 thymoma due to the high rate of AIDs $(13,14,43)$ and should not be delivered in an off-label setting without full disclosure of risks in the multidisciplinary tumor board. Several clinical trials with ICI alone or in combination are ongoing. In Europe, the European Organization for Research and Treatment of Cancer (EORTC) and the European Thoracic Oncology Platform have commenced a phase II study (the NIVOTHYM trial) to assess the efficacy of nivolumab or its combination with ipilimumab in patients with advanced, refractory type B3 thymoma or TC. A strict autoimmune follow-up is planned (NCT03134118). A phase I/II trial with pembrolizumab in TC and thymomas is also underway at MD Anderson Cancer Center (NCT03295227). The National Cancer Institute have designed a phase II trial to assess efficacy and toxicity in thymoma and TC treated with avelumab (NCT03076554). Recently, preliminary results from CAVEATT study (avelumab + axitinib) have been reported with promising outcomes (partial response and stable disease of $40 \%$ and $60 \%$ respectively and, PFS of 7.9 months) and acceptable safety profile (86). Furthermore, combinations of ICI with tyrosine kinase inhibitor (sunitinib or lenvatinib) or with IDO (indoleamina 2,3-dioxigenasa-1) inhibitor (Epacadostat) are under study beside the importance of these pathways in TETs. Of note, no responses have been reported for patients treated with pembrolizumab and epacadoscat combination before study cancellation due to unexpected results reported 
Table 2 Ongoing clinical trials with immune checkpoint inhibitors with or without other agents in TET setting

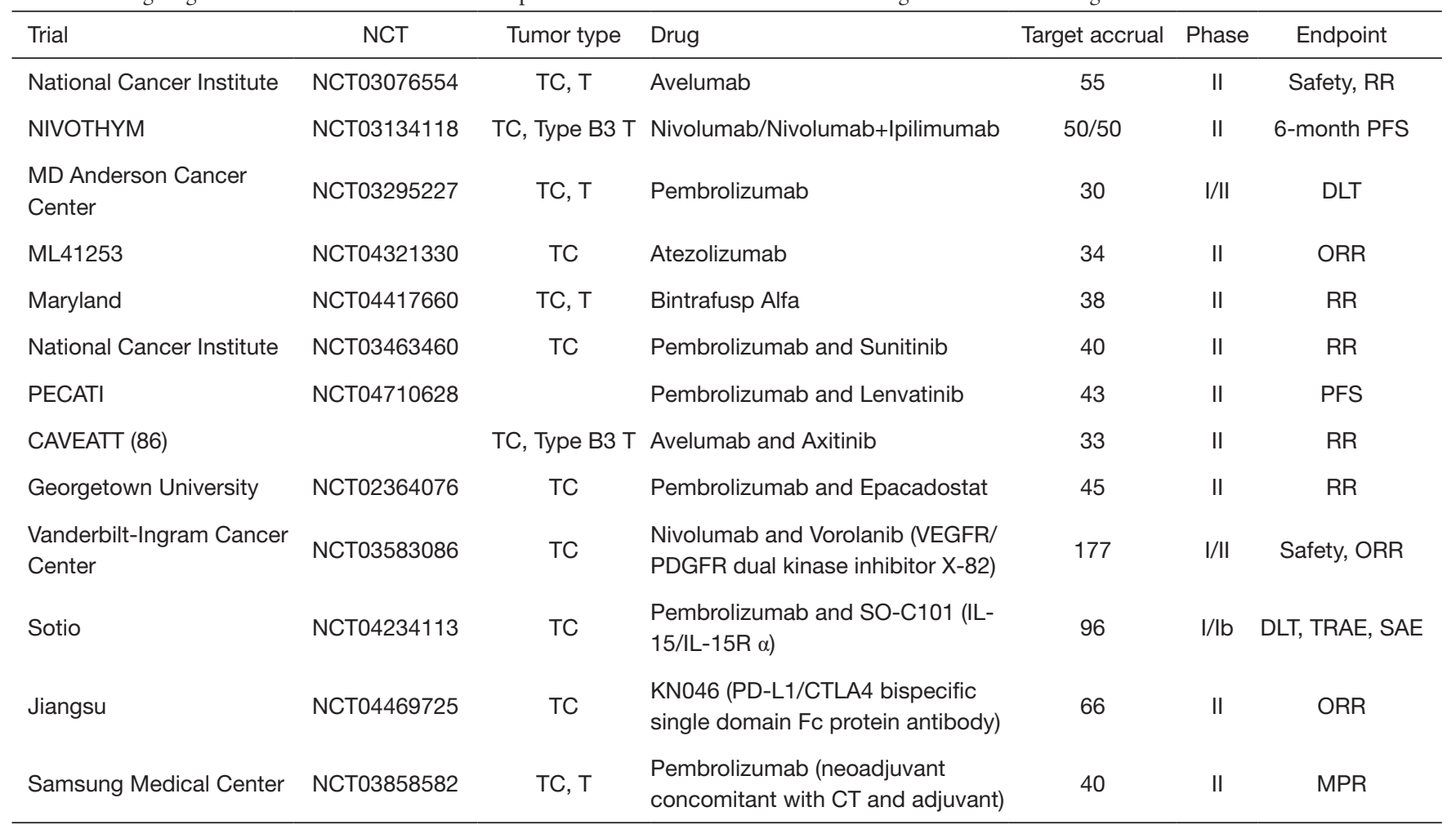

TETs, thymic epithelial tumors; TC, thymic carcinoma; T, thymoma; CT, chemotherapy; RR, response rate; ORR, objective response rate; PFS, progression-free survival; DLT, dose limiting toxicity; TRAE, treatment-related adverse events; SAEs, serious adverse events; MPR, mayor pathology response.

for melanoma $(76,87)$. New explored combinations in agnostic, are also under research in TETs, such as bispecific single domain Fc fusion antibody (PD-L1/CTLA4) KN046 (NCT04469725). Finally, the benefit of ICIs in the neoadjuvant and adjuvant setting in several tumors led to evaluate ICI approaches in TETs as a study with pembrolizumab is exploring (NCT03858582) (Table 2).

\section{Future challenges}

\section{Is it possible to reduce the risk of irAEs in TETs?}

As TETs patients have an increased risk to develop TRAE, it is an important aspect to consider for selection of candidates for ICI treatment. Some strategies to reduce this risk and enhance the safety used of immunotherapy in TET population are needed.

Depending on the histological characteristics for each histological subtype, the probability of developing AIDs is different. Gradation of lymphocyte infiltration differs from B1 (lymphocyte rich) to B3 (lymphocyte poor) (8).
Furthermore, different molecular profiles are related to each histological subtype (22). It has been reported that preexisting autoantibodies against AchR and B cell lymphopenia assessed in thymoma correlates with higher risk of myositis with avelumab (88). In addition, the overexpression of CHRNA1 and RYR3 is present in thymomas with clinical history of MG (22), it has been related with the capacity of tumor cells to secrete mimicry physiological proteins to non-malignant cells (85). A hallmark of these tumors is their association with autoimmunity linked through overexpression of muscle autoantigens and increased aneuploidy (22).

ICIs re-challenges, in patients presenting irAE with the first intent with immunotherapy, have been reported. Some retrospective series have assessed up to $55 \%$ of irAE in this setting but not as severe as the initial treatment (89). However, this scenario has not been well examined in thymoma since the high probability of AIDs in this entity. Tight monitoring and molecular profiling of AIDs in TETs may open an opportunity to incorporate this strategy not to exclude them from clinical trials. It was explored in a study 
with patients with melanoma treated with ipilimumab; of 30 patients with advanced disease and several AIDs (Grave's disease, inflammatory bowel disease or rheumatoid arthritis), $27 \%$ presented exacerbation of AIDs and $33 \%$ reported new irAEs, moreover $50 \%$ of the patients did not reported neither flare nor new irAE (90). Finally, combining ICIs with a selective immunosuppressant have been proposed as preventing flare of AID approach (91), however it may be deeply studied for TETs.

\section{Conclusions}

Immune checkpoint inhibitors may represent a new option and show up new challenges in the field of advanced TETs, although the implementation in the clinical management remains a challenge based on the special biology of thymic cancers. Although the incidence of TRAEs is higher in association with thymoma compared to TC, patients with TC are also at risk of developing immune-related toxicity even during the follow-up; nevertheless, ICI setting may be kept for this entity but requiring a very tight monitoring. New combinations of ICI and targeted therapies are promising. Understanding biological and immune landscape of thymic epithelial cells and the interaction with immune system, is likely the key to prevent autoimmune adverse events of ICIs to be able to find new therapeutic options for this rare disease.

\section{Acknowledgments}

Funding: None.

\section{Footnote}

Provenance and Peer Review: This article was commissioned by the editorial office, Translational Lung Cancer Research for the series "Immunotherapy in other thoracic malignancies and uncommon populations". The article has undergone external peer review.

Reporting Checklist: The authors have completed the narrative review reporting checklist. Available at https:// dx.doi.org/10.21037/tlcr-20-1222

Peer Review File: Available at https://dx.doi.org/10.21037/ tlcr-20-1222

Conflicts of Interest: Both authors have completed the
ICMJE uniform disclosure form (available at https://dx.doi. org/10.21037/tlcr-20-1222). The series "Immunotherapy in other thoracic malignancies and uncommon populations" was commissioned by the editorial office without any funding or sponsorship. B Besse served as the unpaid Guest Editor of the series. Dr. Besse reports grants from Abbvie, Amgen, AstraZeneca, Biogen, Blueprint Medicines, BMS, Celgene, Eli Lilly, GSK, Ignyta, IPSEN, Merck KGaA, MSD, Nektar, Onxeo, Pfizer, Pharma Mar, Sanofi, Spectrum Pharmaceuticals, Takeda, Tiziana Pharma, other from Nerviano, GSK, Pfizer, Roche-Genentech, Lilly, OSE Pharma, MSD, Celgene, Stemcentrx, Ignyta, Abbvie, Loxo Oncology, AstraZeneca, Blueprint Medicines, outside the submitted work. The authors have no other conflicts of interest to declare.

Ethical Statement: The authors are accountable for all aspects of the work in ensuring that questions related to the accuracy or integrity of any part of the work are appropriately investigated and resolved.

Open Access Statement: This is an Open Access article distributed in accordance with the Creative Commons Attribution-NonCommercial-NoDerivs 4.0 International License (CC BY-NC-ND 4.0), which permits the noncommercial replication and distribution of the article with the strict proviso that no changes or edits are made and the original work is properly cited (including links to both the formal publication through the relevant DOI and the license). See: https://creativecommons.org/licenses/by-nc-nd/4.0/.

\section{References}

1. Girard N, Mornex F, Van Houtte P, et al. Thymoma: a focus on current therapeutic management. J Thorac Oncol 2009;4:119-26.

2. Engels EA. Epidemiology of Thymoma and Associated Malignancies. J Thorac Oncol 2010;5:S260-5.

3. Venuta F, Anile M, Diso D, et al. Thymoma and thymic carcinoma. Eur J Cardiothorac Surg 2010;37:13-25.

4. Travis WD, Brambilla E, Burke AP, et al. Introduction to the 2015 World Health Organization Classification of Tumors of the Lung, Pleura, Thymus, and Heart. J Thorac Oncol 2015;10:1240-2.

5. Marx A, Ströbel P, Badve SS, et al. ITMIG Consensus Statement on the Use of the WHO Histological Classification of Thymoma and Thymic Carcinoma: Refined Definitions, Histological Criteria, and Reporting. 
J Thorac Oncol 2014;9:596-611.

6. Ströbel P, Marx A, Zettl A, et al. Thymoma and Thymic Carcinoma: An Update of the WHO Classification 2004. Surg Today 2005;35:805-11.

7. Thomas de Montpréville V, Ghigna MR, Lacroix L, et al. Thymic carcinomas: clinicopathologic study of 37 cases from a single institution. Virchows Arch 2013;462:307-13.

8. Girard N, Shen R, Guo T, et al. Comprehensive genomic analysis reveals clinically relevant molecular distinctions between thymic carcinomas and thymomas. Clin Cancer Res 2009;15:6790-9.

9. Rieker RJ, Muley T, Klein C, et al. An institutional study on thymomas and thymic carcinomas: experience in 77 patients. Thorac Cardiovasc Surg 2008;56:143-7.

10. Masaoka A, Monden Y, Nakahara K, et al. Follow-up study of thymomas with special reference to their clinical stages. Cancer 1981;48:2485-92.

11. Koga K, Matsuno Y, Noguchi M, et al. A review of 79 thymomas: modification of staging system and reappraisal of conventional division into invasive and non-invasive thymoma. Pathol Int 1994;44:359-67.

12. Hishida T, Nomura S, Yano M, et al. Long-term outcome and prognostic factors of surgically treated thymic carcinoma: results of 306 cases from a Japanese Nationwide Database Study. Eur J Cardiothorac Surg 2016;49:835-41.

13. Okumura M, Ohta M, Tateyama H, et al. The World Health Organization histologic classification system reflects the oncologic behavior of thymoma: A clinical study of 273 patients. Cancer 2002;94:624-32.

14. Kondo K, Yoshizawa K, Tsuyuguchi M, et al. WHO histologic classification is a prognostic indicator in thymoma. Ann Thorac Surg 2004;77:1183-8.

15. Meurgey A, Girard N, Merveilleux du Vignaux C, et al. Assessment of the ITMIG Statement on the WHO Histological Classification and of the Eighth TNM Staging of Thymic Epithelial Tumors of a Series of 188 Thymic Epithelial Tumors. J Thorac Oncol 2017;12:1571-81.

16. Girard N, Ruffini E, Marx A, et al. Thymic epithelial tumours: ESMO Clinical Practice Guidelines for diagnosis, treatment and follow-up. Ann Oncol 2015;26:v40-v55.

17. Detterbeck FC, Asamura H, Crowley J, et al. The IASLC/ ITMIG thymic malignancies staging project: development of a stage classification for thymic malignancies. J Thorac Oncol 2013;8:1467-73.

18. Ruffini E, Detterbeck F, van raemdonck D, et al. Tumours of the thymus: A cohort study of prognostic factors from the European Society of Thoracic Surgeons database. Eur
J Cardiothorac Surg 2014;46:361-8.

19. Okuma Y, Saito M, Hosomi Y, et al. Key components of chemotherapy for thymic malignancies: a systematic review and pooled analysis for anthracycline-, carboplatinor cisplatin-based chemotherapy. J Cancer Res Clin Oncol 2015;141:323-31.

20. Thomas A, Rajan A, Berman A, et al. Sunitinib in patients with chemotherapy-refractory thymoma and thymic carcinoma: an open-label phase 2 trial. Lancet Oncol 2015;16:177-86.

21. Zucali PA, De Pas T, Palmieri G, et al. Phase II Study of Everolimus in Patients With Thymoma and Thymic Carcinoma Previously Treated With Cisplatin-Based Chemotherapy. J Clin Oncol 2018;36:342-9.

22. Radovich M, Pickering CR, Felau I, et al. The Integrated Genomic Landscape of Thymic Epithelial Tumors. Cancer Cell 2018;33:244-58.e10.

23. Pan CC, Chen PCH, Chiang H. KIT (CD117) is frequently overexpressed in thymic carcinomas but is absent in thymomas. J Pathol 2004;202:375-81.

24. Wang Y, Thomas A, Lau C, et al. Mutations of epigenetic regulatory genes are common in thymic carcinomas. Sci Rep 2014;4:7336.

25. Mariano C, Ionescu DN, Cheung WY, et al. Thymoma: a population-based study of the management and outcomes for the province of British Columbia. J Thorac Oncol 2013;8:109-17.

26. Topalian SL, Hodi FS, Brahmer JR, et al. Five-Year Survival and Correlates among Patients with Advanced Melanoma, Renal Cell Carcinoma, or Non-Small Cell Lung Cancer Treated with Nivolumab. JAMA Oncol 2019;5:1411-20.

27. Reck M, Rodriguez-Abreu D, Robinson AG, et al. Pembrolizumab versus Chemotherapy for PD-L1Positive Non-Small-Cell Lung Cancer. N Engl J Med 2016;375:1823-33.

28. Powles T, Durán I, van der Heijden MS, et al. Atezolizumab versus chemotherapy in patients with platinum-treated locally advanced or metastatic urothelial carcinoma (IMvigor211): a multicentre, open-label, phase 3 randomised controlled trial. Lancet 2018;391:748-57.

29. Cheng M, Anderson MS. Thymus_torelance_rev_ NatImmu2018. Nat Immunol 2018;19:1.

30. Anderson MS, Su MA. Aire and T cell development. Curr Opin Immunol 2011;23:198-206.

31. Takaba H, Takayanagi H. The Mechanisms of T Cell Selection in the Thymus. Trends Immunol 2017;38:805-16. 
32. Takahama Y. Journey through the thymus: Stromal guides for T-cell development and selection. Nat Rev Immunol 2006;6:127-35.

33. Maverakis E, Goodarzi H, Wehrli LN, et al. The etiology of paraneoplastic autoimmunity. Clin Rev Allergy Immunol 2012;42:135-44.

34. Weksler B, Lu B. Alterations of the immune system in thymic malignancies. J Thorac Oncol 2014;9:S137-42.

35. Kim TK, Herbst RS, Chen L. Defining and Understanding Adaptive Resistance in Cancer Immunotherapy. Trends Immunol 2018;39:624-31.

36. Weissferdt A, Fujimoto J, Kalhor N, et al. Expression of PD-1 and PD-L1 in thymic epithelial neoplasms. Mod Pathol 2017;30:826-33.

37. Lippner EA, Lewis DB, Robinson WH, et al. Paraneoplastic and Therapy-Related Immune Complications in Thymic Malignancies. Curr Treat Options Oncol 2019;20:62.

38. Shelly S, Agmon-Levin N, Altman A, et al. Thymoma and autoimmunity. Cell Mol Immunol 2011;8:199-202.

39. Huang J, Ahmad U, Antonicelli A, et al. Development of the International Thymic Malignancy Interest Group International Database: An unprecedented resource for the study of a rare group of tumors. J Thorac Oncol 2014;9:1573-8.

40. Boucher M, Dansin E, Kerjouan M, et al. OA 03.01 Prevalence of Autoimmune Diseases in Thymic Epithelial Tumors (TET) Insights from RYTHMIC. J Thorac Oncol 2017;12:S1748-9.

41. Noël N, Le Roy A, Hot A, et al. Systemic lupus erythematosus associated with thymoma: A fifteenyear observational study in France. Autoimmun Rev 2020;19:102464.

42. Tormoehlen LM, Pascuzzi RM. Thymoma, Myasthenia Gravis, and Other Paraneoplastic Syndromes. Hematol Oncol Clin North Am 2008;22:509-26.

43. Benitez J, Boucher M, Dansin E, et al. Prevalence of autoimmune diseases in Thymic Epithelial Tumors (TET) insights from RYTHMIC. In: ASCO Virtual Congress 2020. 2020.

44. Benitez J, Boucher M, Dansin E, et al. Studying autoimmune diseases with Thymic Epithelial Tumors (TET): real-world insight from RYTHMIC. ESMO Immuno-Oncology Congr. 2020.

45. Facchinetti F, Marabelle A, Rossi G, et al. Moving immune checkpoint blockade in thoracic tumors beyond NSCLC. J Thorac Oncol 2016;11:1819-36.

46. Nakajima J, Okumura M, Yano M, et al. Myasthenia gravis with thymic epithelial tumour: a retrospective analysis of a Japanese database. Eur J Cardiothorac Surg 2016;49:1510-5.

47. Molina T, Bluthgen MV, Chalabreysse L, et al. Pathological central review of 290 thymic epithelial tumors (TET): The national network RYTHMIC experience. J Clin Oncol 2016;34:8568.

48. Gilhus NE. Myasthenia gravis. N Engl J Med 2016;375:2570-81.

49. Luo J, Lindstrom J. Myasthenogenicity of the main immunogenic region and endogenous muscle nicotinic acetylcholine receptors. Autoimmunity 2012;45:245-52.

50. Liu Y, Zhang H, Zhang P, et al. Autoimmune regulator expression in thymomas with or without autoimmune disease. Immunol Lett 2014;161:50-6.

51. Scarpino S, Di Napoli A, Stoppacciaro A, et al. Expression of autoimmune regulator gene (AIRE) and T regulatory cells in human thymomas. Clin Exp Immunol 2007;149:504-12.

52. Guo F, Wang CY, Wang S, et al. Alteration in gene expression profile of thymomas with or without myasthenia gravis linked with the nuclear factor-kappaB/autoimmune regulator pathway to myasthenia gravis pathogenesis. Thorac Cancer 2019;10:564-70.

53. Schultz A, Hoffacker V, Wilisch A, et al. Neurofilament is an autoantigenic determinant in myasthenia gravis. Ann Neurol 1999;46:167-75.

54. Marx A, Wilisch A, Schultz A, et al. Expression of neurofilaments and of a titin epitope in thymic epithelial tumors. Implications for the pathogenesis of Myasthenia gravis. Am J Pathol 1996;148:1839-50.

55. Benitez J, Job B, Thomas de Montpréville V, et al. Cancer activation pathways of Thymic Epithelial Tumors (TETs) by targeted gene expression analysis. ASCO virtual Congr 2021. 2021.

56. Ruan X, Lu X, Gao J, et al. Multiomics data reveals the influences of myasthenia gravis on thymoma and its precision treatment. J Cell Physiol 2021;236:1214-27.

57. Petrini I, Meltzer PS, Kim IK, et al. A specific missense mutation in GTF2I occurs at high frequency in thymic epithelial tumors. Nat Genet 2014;46:844-9.

58. Giaccone G, Kim C, Thompson J, et al. Pembrolizumab in patients with thymic carcinoma: a single-arm, singlecentre, phase 2 study. Lancet Oncol 2018;19:347-55.

59. Saito M, Fujiwara Y, Asao T, et al. The genomic and epigenomic landscape in thymic carcinoma. Carcinogenesis 2017;38:1084-91.

60. Lee MC, Hsiao TH, Chuang HN, et al. Molecular 
profiling of thymoma with myasthenia gravis: Risk factors of developing myasthenia gravis in thymoma patients.

Lung Cancer 2020;139:157-64.

61. Broadley J, Wesselingh R, Seneviratne U, et al. Peripheral Immune Cell Ratios and Clinical Outcomes in Seropositive Autoimmune Encephalitis: A Study by the Australian Autoimmune Encephalitis Consortium. Front Immunol 2021;11:597858.

62. Pinal-Fernandez I, Casal-Dominguez M, Derfoul A, et al. Machine learning algorithms reveal unique gene expression profiles in muscle biopsies from patients with different types of myositis. Ann Rheum Dis 2020;79:1234-42.

63. Havel JJ, Chowell D, Chan TA. The evolving landscape of biomarkers for checkpoint inhibitor immunotherapy. Nat Rev Cancer 2019;19:133-50.

64. Goodman AM, Kato S, Bazhenova L, et al. Tumor mutational burden as an independent predictor of response to immunotherapy in diverse cancers. Mol Cancer Ther 2017;16:2598-608.

65. Wolf Y, Bartok O, Patkar S, et al. UVB-Induced Tumor Heterogeneity Diminishes Immune Response in Melanoma. Cell 2019;179:219-35.e21.

66. Rizvi NA, Hellmann MD, Snyder A, et al. Mutational landscape determines sensitivity to PD-1 blockade in nonsmall cell lung cancer. Science 2015;348:124-8.

67. Marchevsky AM, Walts AE. PD-L1, PD-1, CD4, and CD8 expression in neoplastic and nonneoplastic thymus. Hum Pathol 2017;60:16-23.

68. Wei YF, Chu CY, Chang CC, et al. Different pattern of PD-L1, IDO, and FOXP3 Tregs expression with survival in thymoma and thymic carcinoma. Lung Cancer 2018;125:35-42.

69. Katsuya Y, Fujita Y, Horinouchi H, et al. Immunohistochemical status of PD-L1 in thymoma and thymic carcinoma. Lung Cancer 2015;88:154-9.

70. Padda SK, Riess JW, Schwartz EJ, et al. Diffuse high intensity PD-L1 staining in thymic epithelial tumors. J Thorac Oncol 2015;10:500-8.

71. Arbour KC, Naidoo J, Steele KE, et al. Expression of PD-L1 and other immunotherapeutic targets in thymic epithelial tumors. PLoS One 2017;12:e0182665.

72. Yokoyama S, Miyoshi H, Nakashima K, et al. Prognostic value of programmed death ligand 1 and programmed death 1 expression in thymic carcinoma. Clin Cancer Res 2016;22:4727-34.

73. Higuchi R, Goto T, Hirotsu Y, et al. PD-L1 Expression and Tumor-Infiltrating Lymphocytes in Thymic Epithelial
Neoplasms. J Clin Med 2019;8:1833.

74. Yokoyama S, Miyoshi H, Nishi T, et al. Clinicopathologic and Prognostic Implications of Programmed Death Ligand 1 Expression in Thymoma. Ann Thorac Surg 2016;101:1361-9.

75. Katsuya Y, Horinouchi H, Asao T, et al. Expression of programmed death 1 (PD-1) and its ligand (PD-L1) in thymic epithelial tumors: Impact on treatment efficacy and alteration in expression after chemotherapy. Lung Cancer 2016;99:4-10.

76. Giaccone G, Kim C. Durable Response in Patients With Thymic Carcinoma Treated With Pembrolizumab After Prolonged Follow-Up. J Thorac Oncol 2021;16:483-5.

77. Cho J, Kim HS, Ku BM, et al. Pembrolizumab for Patients With Refractory or Relapsed Thymic Epithelial Tumor: An Open-Label Phase II Trial. J Clin Oncol 2019;37:2162-70.

78. Katsuya Y, Horinouchi H, Seto T, et al. Single-arm, multicentre, phase II trial of nivolumab for unresectable or recurrent thymic carcinoma: PRIMER study. Eur J Cancer 2019;113:78-86.

79. Heery CR, O’Sullivan-Coyne G, Madan RA, et al. Avelumab for metastatic or locally advanced previously treated solid tumours (JAVELIN Solid Tumor): a phase 1a, multicohort, dose-escalation trial. Lancet Oncol 2017;18:587-98.

80. Rajan A, Heery CR, Perry S, et al. Safety and clinical activity of anti-programmed death-ligand 1 (PD-L1) antibody (ab) avelumab (MSB0010718C) in advanced thymic epithelial tumors (TETs). J Clin Oncol 2016;34:e20106.

81. Kumar V, Chaudhary N, Garg M, et al. Current Diagnosis and Management of Immune Related Adverse Events (irAEs) Induced by Immune Checkpoint Inhibitor Therapy. Front Pharmacol 2017;8:49.

82. Chen Q, Huang DS, Zhang LW, et al. Fatal myocarditis and rhabdomyolysis induced by nivolumab during the treatment of type B3 thymoma. Clin Toxicol (Phila) 2018;56:667-71.

83. So H, Ikeguchi R, Kobayashi M, et al. PD-1 inhibitorassociated severe myasthenia gravis with necrotizing myopathy and myocarditis. J Neurol Sci 2019;399:97-100.

84. Touat M, Maisonobe T, Knauss S, et al. Immune checkpoint inhibitor-related myositis and myocarditis in patients with cancer. Neurology 2018;91:e985-94.

85. Takamori M. Myasthenia Gravis: From the Viewpoint of Pathogenicity Focusing on Acetylcholine Receptor Clustering, Trans-Synaptic Homeostasis and Synaptic 
Stability. Front Mol Neurosci 2020;13:86.

86. Conforti F, Pala L, Catania C, et al. Safety and activity of Combined AVElumab with Axitinib in unresectable or metastatic Thymomas B3 and Thymic carcinomas: The CAVEATT study. J Clin Oncol 2020;38:e21114.

87. Long GV, Dummer R, Hamid O, et al. Epacadostat plus pembrolizumab versus placebo plus pembrolizumab in patients with unresectable or metastatic melanoma (ECHO-301/KEYNOTE-252): a phase 3, randomised, double-blind study. Lancet Oncol 2019;20:1083-97.

88. Mammen AL, Rajan A, Pak K, et al. Pre-existing antiacetylcholine receptor autoantibodies and $\mathrm{B}$ cell lymphopaenia are associated with the development of myositis in patients with thymoma treated with avelumab, an immune checkpoint inhibitor targeting programmed death-ligand 1. Ann Rheum Dis 2019;78:150-2.

89. Simonaggio A, Michot JM, Voisin AL, et al. Evaluation of Readministration of Immune Checkpoint Inhibitors After Immune-Related Adverse Events in Patients With Cancer. JAMA Oncol 2019;5:1310-7.

90. Johnson DB, Sullivan RJ, Ott PA, et al. Ipilimumab Therapy in Patients With Advanced Melanoma and Preexisting Autoimmune Disorders. JAMA Oncol 2016;2:234-40.

91. Haanen J, Ernstoff MS, Wang Y, et al. Autoimmune diseases and immune-checkpoint inhibitors for cancer therapy: review of the literature and personalized riskbased prevention strategy. Ann Oncol 2020;31:724-44.

Cite this article as: Benitez JC, Besse B. Narrative review of immunotherapy in thymic malignancies. Transl Lung Cancer Res 2021;10(6):3001-3013. doi: 10.21037/tlcr-20-1222 